\title{
An Emerging Method of the Non-contact Displacement Measurement by
}

\section{Video} \author{
Jichen Ma ${ }^{1, a}$ Junjiang Xiao ${ }^{2, b}$ Ruolei Liu ${ }^{3, c}$ Jinlong Zhu ${ }^{4, d}$ \\ ${ }^{1}$ Department of Engineering Mechanics, School of Aerospace Engineering and Applied Mechanics, \\ Tongji University, Shanghai, China, 200092 \\ ${ }^{2}$ Department of Engineering Mechanics, School of Aerospace Engineering and Applied Mechanics, \\ Tongji University, Shanghai, China, 200092 \\ ${ }^{3}$ Department of Engineering Mechanics, School of Aerospace Engineering and Applied Mechanics, \\ Tongji University, Shanghai, China, 200092 \\ ${ }^{4}$ Senior Experimentalist, School of Aerospace Engineering and Applied Mechanics, Tongji \\ University, Shanghai, China, 200092 \\ a973028484@qq.com b1353644@tongji.edu.cn 'c645587436@qq.com \\ Iongjinzhu@tongji.edu.cn
}

Keywords: the Video Displacement non-contact Measuring System; the arithmetic matching video's local back-projection images; target tracking; common film equipment

Abstract: It is demonstrated an emerging measuring method of the Video Displacement Non-contact Measuring System. Through cameras and camcorders in mobile phones, the algorithm based on histogram characteristic of images is built and optimized in computing methods, obtaining displacement and deflection of the low-frequency-vibration structure.

\section{Introduction}

The displacement observation is the most basic and important part of engineering structure health monitoring. The displacement-observation method learns operating states of the structure and component under certain loads through measuring their deformations.

As an emerging method of non-contact measuring, the non-contact video displacement measuring system has great advantages compared with traditional contact-type measuring methods. It is able to accurately measure the structures in vibration, with high-level automaticity. We discuss about the designing of the algorithm based on statistical characteristics of video's images, from common digital devices with the function of video recording, such as cameras and smartphones. Then we optimize the algorithm, and verify it through engineering-project cases.

\section{The design of video displacement measuring system using common digital photographic devices}

The video displacement measuring system consists of imaging system, computing system and software. The processes include video capture form cameras or smartphones, putting the video data into computer, dealing with the data through the algorithm and getting output. Tracking the mark points on object structure, superposing each micro line blocks of displacement, we get displacement curves of the whole process. Steps are shown as Fig.1: 


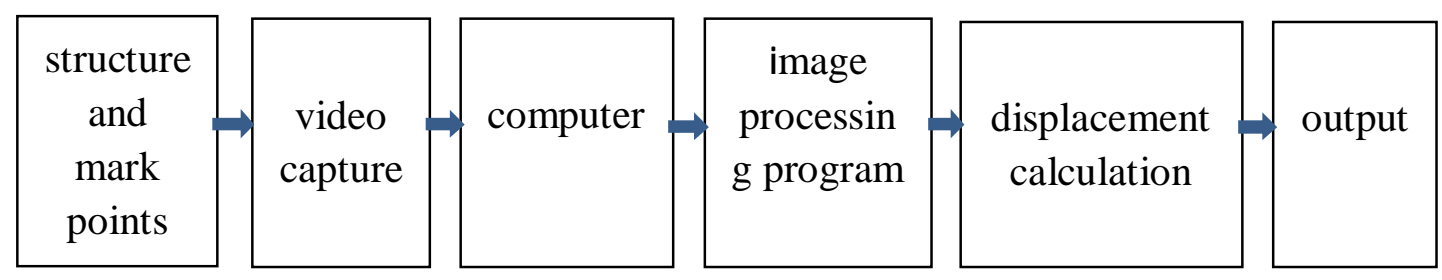

Figure 1. The whole process of project

\section{The method of video displacement measuring using common digital photographic devices}

\section{The algorithm based on statistical characteristics of video's images}

Image detections and target tracking are important subjects in the fields of video content analysis and computer vision, and correlation techniques are widely used. Specifically, it means cutting the object's motion regions from each image in video's image sequences, and tracks the objects among consecutive frames of the whole video. The algorithmic process we decided is shown in Fig.2

In view of the requirement of displacement measuring in engineering testing, it is necessary to track the object or mark point continuously, and get the data of displacement automatically. There are deformations in engineering structure during process of sport, and the entirety can't be seen in rigid motion. So it is null to match the object region with the template simply. As a basic method of estimation without parameters, the histogram statistics method doesn't impose any assumption about the data distribution. The color histogram of the target area is regarded as the feature, with rotation invariance, and not affected by the change of the target's size and shape. And it is known as the back-projection of images that the original video images are changed into color probability distribution images by color histograms.

Set $\left\{\boldsymbol{x}_{\boldsymbol{i}}\right\}(\boldsymbol{i}=1,2, \ldots, \boldsymbol{n})$ as a point set of the target area, the $\boldsymbol{m}$ as the color resolution, $\boldsymbol{q}_{\boldsymbol{u}}$ $(\boldsymbol{u}=\mathbf{1}, 2, \ldots \boldsymbol{m})$ as the probability of color in the target group. Now a mapping, $\boldsymbol{c}: \boldsymbol{R} \rightarrow\{\mathbf{1}, \mathbf{2}, \ldots, \boldsymbol{m}\}$, is defined, which means that for each pixel point $\boldsymbol{x}_{\boldsymbol{i}}$, and the index value of its color $\boldsymbol{c}\left(\boldsymbol{x}_{i}\right)$, the probability of each color value arising in the target area is ${ }^{[1]}$ :

$$
\mathrm{q}_{\mathrm{u}}=\sum_{\mathrm{i}=1}^{\mathrm{n}} \delta\left[\mathrm{c}\left(\mathrm{x}_{\mathrm{i}}\right)-\mathrm{u}\right]
$$

And the $\boldsymbol{\delta}$ is Function Kornecker.

In practical engineering tests, we find that in RGB (Red, Green, Blue) color space, the target area in general structures changes non-significantly. And in HSV (Hue, Saturation, Value) space, because the influence of illumination in short time is puny, it is not necessary to use $\mathrm{H}$ component deliberately as the feature to reduce the illumination effect. Now, usual mechanism of images is RGB, and it will affect images quality to change RGB images into HSV images, especially when the saturation and brightness are small. So choosing grayscale as the characteristic can be more convenient and concise. In this case, $\boldsymbol{m}=256$. 


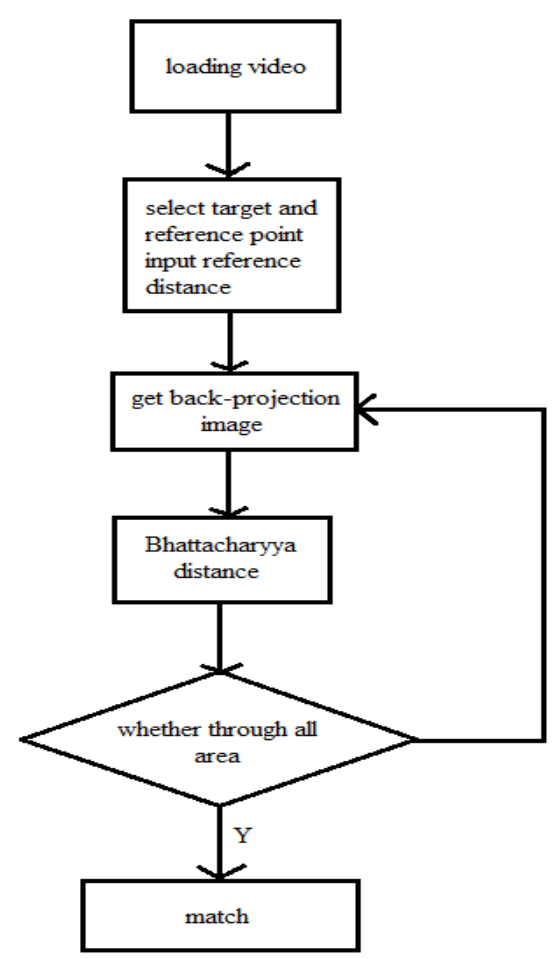

Figure 2. The algorithmic process

In order to complete the match, we have the similarity measure. As for $\boldsymbol{q}_{\boldsymbol{u}}$, we do the cluster analysis as follow. The amount of samples (in area matched) is $\boldsymbol{n}$, and each sample has $\boldsymbol{m}$ indicators, so the primary information matrix $\boldsymbol{H}_{i}$ is ${ }^{[2]}$

$$
\mathrm{H}_{\mathrm{i}}=\left[\begin{array}{ccc}
\mathrm{h}_{1} & \mathrm{~h}_{2} & \mathbf{h}_{\mathrm{p}} \\
\mathrm{h}_{\mathrm{p}+1} & \mathrm{~h}_{\mathrm{p} 2} & \mathrm{~h}_{\mathrm{p}+3} \\
\cdot & - & \cdot \\
- & - & \cdot \\
\cdot & \cdot & \cdot \\
\mathrm{h}_{\mathrm{m}-2} & \mathrm{~h}_{\mathrm{m}-1} & \mathrm{~h}_{\mathrm{m}}
\end{array}\right]
$$

The component $\boldsymbol{h}_{\boldsymbol{j}}$ is the observation of the $\boldsymbol{j}$-th index of the $\boldsymbol{H}_{\boldsymbol{i}}$-th sample. We use $\boldsymbol{h}_{\boldsymbol{1}}, \boldsymbol{h}_{\mathbf{2}}, \ldots, \boldsymbol{h}_{\boldsymbol{m}}$ to express the indexes, and use $\boldsymbol{H}_{1}, \boldsymbol{H}_{2}, \ldots, \boldsymbol{H}_{n}$ to express the samples. If we regard the indexes as points in hyperspace, the proximity between the two samples can be measured by Bhattacharyya distance:

$$
\mathrm{D}(\mathrm{x})=\Sigma \sqrt{\mathrm{H}_{i}(\mathrm{x}) * \mathrm{H}_{\mathrm{j}}(\mathrm{x})}
$$

Using above methods, the program deals with the characteristic-value histograms of the target areas in the initial frame and subsequent frames, to calculate the similarity of two regions. The value $\boldsymbol{D}$ is between $\mathbf{0}$ and $\mathbf{1}$. The greater the value $\boldsymbol{D}$ is, the more similar two models will be. And candidate models calculated in the candidate areas of the current frame make the region with the greatest value $\boldsymbol{D}$ to be the target position in this frame.

In order to make it more accurate to capture the target, and to be more sensitive to the change 
rate, we can make the gradient of $\boldsymbol{D}(\boldsymbol{x})$ in the target area bigger, and reduce the gradient on the edge of area. So we alter the formula to:

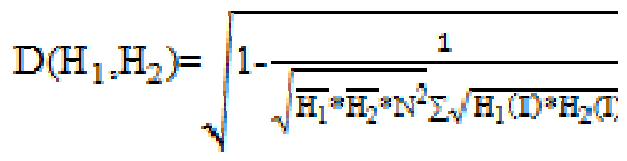

Due to the central pixel in matching area is less and objective pixel in the peripheral may be obscured or affected by background, it's less reliable. We give objective pixel at different location different weights. The closer distance with the center, the greater weights it has. We introduce kernel density estimation into objective function, kernel density estimation is:

$$
A=\frac{1}{\left(x_{i}-x_{0}\right)^{2}+\left(y_{1}-y_{0}\right)^{2}+1}
$$

And

$$
\mathrm{D}\left(\mathrm{H}_{1}, \mathrm{H}_{2}\right)=\mathrm{A} * \sqrt{1-\frac{1}{\sqrt{\sqrt{\mathrm{H}_{1}} * \overline{\mathrm{H}_{2}} * \mathrm{~N}^{2}} \Sigma \sqrt{\mathrm{H}_{1}(\mathrm{D})^{*} \mathrm{H}_{2}(1)}}}
$$

The revised $\boldsymbol{D}$ value between $\mathbf{0}$ to $\mathbf{l}$. The smaller quantitative value of $\boldsymbol{D}$, the more similar the two models. The model is calculated in different area in the current frame and which makes $\boldsymbol{D}$ least is objective location.

\section{Calculation's optimization based on histogram}

In the calculation of statistical histogram of pixel's characteristic value if we use simple enumerative statistical method, we will conduct a large number of redundant computations. What needs to calculate the histogram of the area is not the whole picture. Even we calculate the pixel statistics of entire image which use each pixel as the center of many sub area and get the reverse projection values of all the points to the template according to it, eventually what we need to conduct density calculation and matching area is not the whole picture. Because in the practical engineering problems, the speed of target object which we process will not show high speed or ultra-high speed. In other words, in the limit which the camera equipment reflect on tiny time, meaning the limit between two frames, the target object is presumed to be none of large displacement far ahead of the scale itself. If there is such large displacement, there are only two possible situations. At first it is the photographic equipment conditions. May the frame rate of video equipment is not enough. In the case we cannot assure the quality of the video and we should consider the influence and restriction factor of hardware condition. At the second the Camera device is good but the speed of moving objects is too fast. There is a large displacement between frames .In this case namely before the moving object does not meet the assumption of small displacement between the two frames, the picture has become blurred because of the larger displacement in the exposure time. This is beyond the design requirements of the new algorithm in theory.

So we need to conduct related assumptions before optimization. Motion image analysis based on good and stable target image. Stability means that the object movement image will not appear to 'jump' between frames due to large sampling frequency. Specifically we assume that displacement 
does not exceed $50 \%$ of its size. Of course, if the sampling frequency is insufficient

The assumed displacement ratio can appropriately expand. At the same time, calculation of the algorithm will increase. So in the straight side statistics of pixel characteristic and generating process of reverse projection we just calculate the remaining region. The size of this area is only related to the size of the sample template. As a result the complexity of the algorithm reduce from $\boldsymbol{O}(\boldsymbol{M N H W})$ to $\boldsymbol{O}(\boldsymbol{H} 2 \boldsymbol{W} 2)$. (M, $\boldsymbol{N}$ is full size, $\boldsymbol{H}, \boldsymbol{W}$ is target size)

There will be a lot of redundant enumeration when we use enumeration statistical method to calculate statistical histogram. If we calculate the pixel statistic histogram of area which centered on each point. Obviously there are lots of overlaps between the adjacent two points. And its repeated enumeration and statistics to the overlap that simple enumeration statistical algorithms are inefficient. Through adduce basic ideas of dynamic programming, we can replace by simple enumeration calculation state function. After preprocessing the whole calculation area and obtaining all the state function, we can calculate the pixel statistic data of each point and avoid all the redundant enumeration statistics.

We find a point on the boundary angle in computing area as a 'reference point'.(the algorithm use the upper left corner point as example) We use the state function $\boldsymbol{f}(\boldsymbol{x}, \boldsymbol{y}, \boldsymbol{k})$ to express the area of the rectangle, from the reference point to point $(x, y)$ (reference point for the upper left corner and point $(\boldsymbol{x}, \boldsymbol{y})$ for the lower right corner), $\boldsymbol{k}$ pixel as pixel features(gray level as pixel features, so $\boldsymbol{k}$ ranges for $[0,255])$. Using the principle of the class we can draw state transfer equation function $f$ :

$$
f(x, y, k)=\left\{\begin{array}{c}
f(x-1, y, k)+f(x, y-1, k)-f(x-1, y-1, k), k \neq I(x, y) \\
f(x-1, y, k)+f(x, y-1, k)-f(x-1, y-1, k)+1, k=1(x, y)
\end{array} ; x_{0}, y>y_{0}\right.
$$

Among them $\boldsymbol{x}_{\boldsymbol{0}}, \boldsymbol{y}_{\boldsymbol{0}}$ are reference point coordinates, (the algorithm based on the upper left corner for the direction of the origin of coordinates) transfer equation for the border is:

$$
\begin{aligned}
& f\left(x_{0}, y\right)=\left\{\begin{array}{c}
f\left(x_{0}, y-1, k\right), k \neq I\left(x_{0} y\right) \\
f\left(x_{0} y-1, k\right)+1, k=I\left(x_{0} y\right)
\end{array}, y>y_{0}\right. \\
& f\left(x, y_{0}, k\right)=\left\{\begin{array}{c}
f\left(x-1, y_{0}, k\right), k \neq I\left(x_{0} y_{0}\right) \\
f\left(x-1, y_{0}, k\right)+1, k=I\left(x_{2} y_{0}\right)
\end{array}, x>x_{0}\right.
\end{aligned}
$$

Benchmark for boundary conditions is

$$
f\left(x_{0}, y_{0}, k\right)=\left\{\begin{array}{l}
0, k \neq I\left(x_{0}, y_{0}\right) \\
1, k=I\left(x_{0}, y_{0}\right)
\end{array}\right.
$$

We can see that the state function can be linearly calculated in sequence. Therefore, complexity of preprocessing algorithm is $\boldsymbol{O}(\boldsymbol{H W K})$. After the pretreatment of all the state function, as long as using the recycling area class theory, we can quickly calculate all target eigenvalue statistical histogram in constant complexity algorithm. So the complexity of the algorithm is reduced to $\boldsymbol{O}(\boldsymbol{H W K})$.

\section{Experimental Analysis And Conclusion}

We use mechanical vibration table in experiment center of Tongji University as the standard source, waveform is sine wave, vibration waveform frequency is $2 \mathrm{~Hz}$, and total amplitude is $\mathbf{1 0 \mathbf { m m }}$. We 
choose D7100 Nikon camera and video image sequence is a standard 1080p (1920*1080). Mobile phones video displacement measured by $\mathrm{Mi}-4 \mathrm{c}$ and video image sequence is a standard 720p $(1080$ $x 720$ ). We determine the distance between experiment and digital camera, the digital camera focal length, the influence of environment light and mobile phone camera processing on the experimental results.

In fig.3, we list six groups of displacement-time curve in different experimental conditions. The experimental results are listed in TABLE I:

TABLE I: THE EXPERIMENTAL DATA UNDER DIFFERENT CONDITIONS

\begin{tabular}{|r|r|r|r|r|r|r|}
\hline Order & Equipment & Interval[m] & $\begin{array}{c}\text { Focal } \\
\text { length[mm] }\end{array}$ & Light & $\begin{array}{c}\text { Absolute } \\
\text { error [mm] }\end{array}$ & $\begin{array}{c}\text { Relative } \\
\text { error [\%] }\end{array}$ \\
\hline a & $\begin{array}{c}\text { Digital } \\
\text { camera }\end{array}$ & 1 & 17 & Nature & 0.373 & 3.73 \\
\hline $\mathrm{b}$ & $\begin{array}{c}\text { Digital } \\
\text { camera }\end{array}$ & 1 & 50 & Nature & 0.064 & 0.64 \\
\hline $\mathrm{c}$ & $\begin{array}{c}\text { Digital } \\
\text { camera }\end{array}$ & 1 & 17 & Bright & 0.132 & 1.32 \\
\hline $\mathrm{d}$ & $\begin{array}{c}\text { Digital } \\
\text { camera }\end{array}$ & 1 & 50 & Bright & 0.108 & 1.08 \\
\hline $\mathrm{f}$ & $\begin{array}{c}\text { Digital } \\
\text { camera }\end{array}$ & 5 & 210 & Nature & 0.113 & 1.13 \\
\hline Phone & 1 & 1 & Nature & 0.395 & 3.95 \\
\hline
\end{tabular}

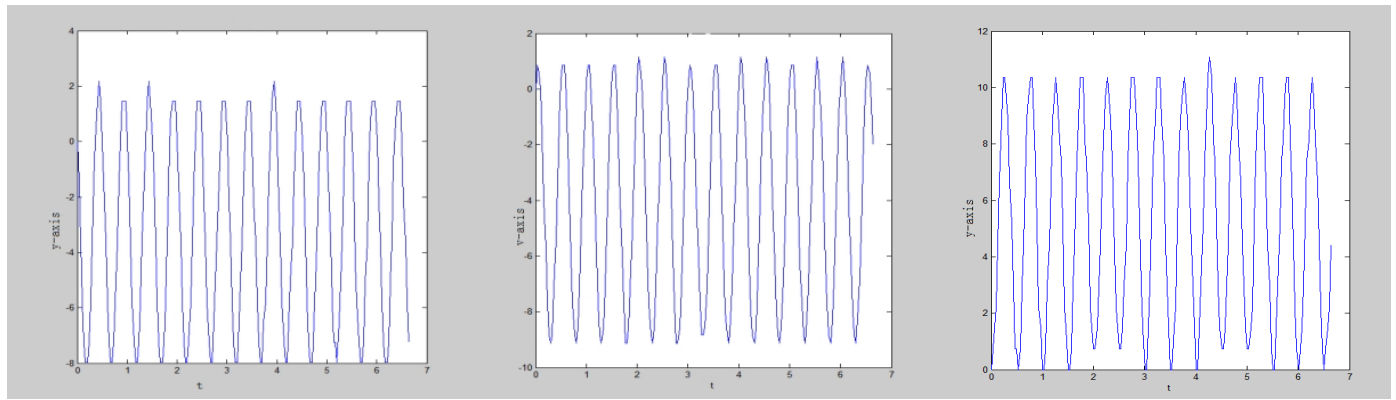

a

b

c 


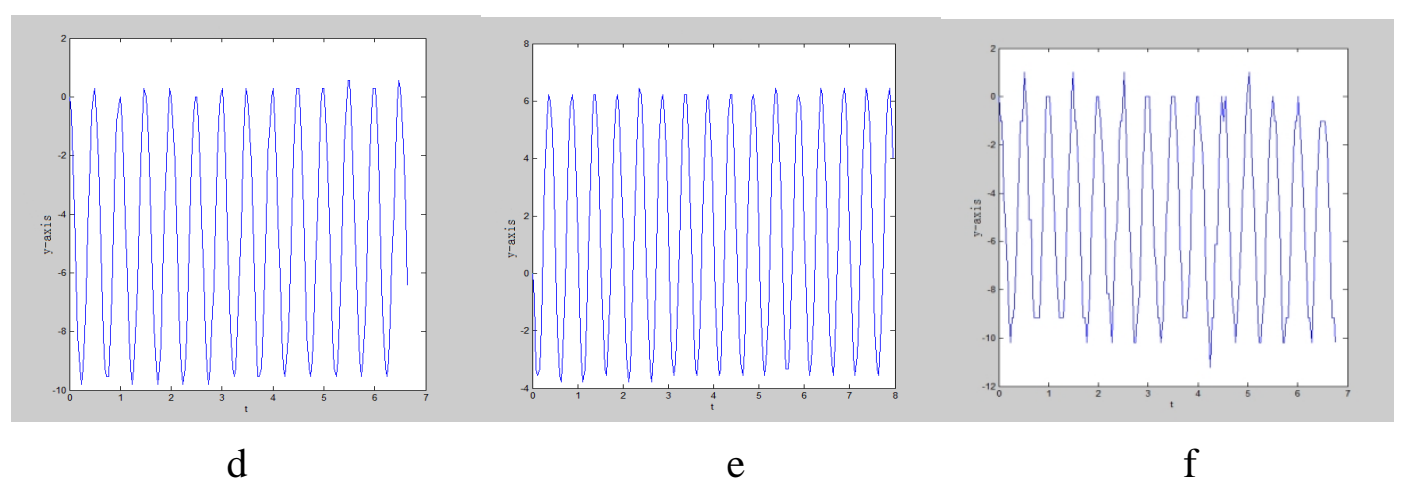

Figure 3. Displacement-time curve in different experimental conditions

\section{Conclusion:}

(1)On the premise of same experimental environment and guaranteeing the complete image clear, closer distance between digital camera and the object, longer digital camera focal length, the capture effect become well and error of the displacement curve become smaller.

We use the unit pixel's actual distance dis to measure difference:

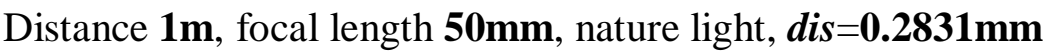

Distance $\mathbf{1 m}$, focal length $\mathbf{1 7} \mathbf{m m}$, nature light, dis $=\mathbf{0 . 7 4 9 6} \mathbf{m m}$

Distance $5 \mathrm{~m}$, focal length $210 \mathrm{~mm}$, nature light, $\boldsymbol{d i s}=\mathbf{0 . 2 2 2 8 \mathrm { mm }}$

Comparing experimental results, larger dis, poorer capture effect. Error is larger. In view of the long distance, in order to improve the dis value, we can change the telephoto lens and expand the selected target area.

(2)Light has little effect on the experimental results.

(3)Mobile acquisition can meet the requirements of close displacement measurement.

\section{Complimentary Close}

Displacement of video monitoring has great engineering application value and commercial potential, especially discussed in this paper, using common digital camera or mobile devices to measure the deflection displacement of low frequency. It can greatly reduce the cost and the user does not need to purchase expensive equipment to achieve measurement. We hope that there is new development and progress in this field.

\section{Acknowledgements}

At the point of finishing this paper, we would like to express our sincere thanks to the Mutual Fund of National Undergraduate Training Program for Innovation and Entrepreneurship giving us financial support. Also thanks for National Mechanics Experimental Teaching Demonstration Center of Tongji University providing laboratory equipment and essential help.

\section{References}

[1] Li jing, Junzheng Wang. Image detection and target tracking technology. Beijing: Beijing Institute of Technology Press, 2014.4 (In Chinese)

[2] Decun Zhang. Statistics (second edition). Beijing: science press, 2009 (In Chinese) 
[3] Zhou xiang, Yihong Liu. Engineering structure detection. Beijing: Peking University press (In Chinese)

[4] Huixuan Gao. Practical statistical method and SAS system. Beijing: Peking University press, 2009.10 (In Chinese)

[5] Zhao qing. CamShift tracking algorithm based on texture information research: (master thesis). Tianjin: nankai university, 2012. (In Chinese)

[6] Liu yi. Partial CamShift tracking algorithm: (undergraduate dissertation). Wuhan: wuhan university of technology, 2011. (In Chinese)

[7] K.Fukunaga and L.D.Hostetler,"The Estimation of the Gradient of a Density Function,with Applications in Pattern Recognition" IEEE Trans.Information Theory,vol.21,pp.32-40,1975.

[8] Shucui Lu, Huimin Wang, Yanhua Wang. Moving target tracking study based on Mean Shift algorithm [J]. Guangdong Communication Technology, 2014, 34 (9) : 23-26. (In Chinese)

[9] Zhihong Xi, Yongjia Li,Duan lian. The target tracking algorithm study based on local features and Mean Shift [J]. Electronic Science and Technology, 2015, 28 (6) : 13-16. (In Chinese)

[10] Shijun Hu, Hongxiang Zhang, Dailu Zhang etc. Non-contact video extensometer used in the mechanical properties test [J]. Science and Technology Review, 2012, 30 (19) : 60-62. (In Chinese)

[11] Zhanfu Wang, Liping Xie. Non-contact video strain measurement system design [J]. Tool Engineering, 2011, 45 (8) : 91-94. (In Chinese)

[12] Li yang, The moving target tracking algorithm based on video sequence [J]. Electronic Science and Technology, 2012, 25 (8) : 125-127. (In Chinese)

[13] Minghao Hu, Mingwu Ren,Jingyu Yang. A real-time tracking of moving objects based on histogram model algorithm [J]. Computer engineering and applications, 2004, 40 (3) : 71-74. (In Chinese)

[14]Ghassabeh Y A. A sufficient condition for the convergence of the mean shift algorithm with Gaussian kernel[J]. Journal of Multivariate Analysis, 2015, 135:1-10. 